\title{
Tau suppresses neuronal activity in vivo, even before tangles form
}

\section{Marc Aurel Busche, UK Dementia Research Institute, University College London, UK}

\author{
contact: m.busche@ucl.ac.uk
}

A number of neurodegenerative disorders including Alzheimer's disease, progressive supranuclear palsy (PSP) and some forms of frontotemporal dementia (FTD) are associated with the build-up of abnormal tau proteins inside neurons. In the healthy brain, tau is located mainly in axons, but in tauopathies the protein becomes hyperphosphorylated and accumulates in the form of intraneuronal aggregates called neurofibrillary tangles. Tangles are associated with neuronal loss and cognitive impairment in patients, but there is also increasing evidence that tangles may not be acutely toxic and can persist inside intact neurons for at least several months. For example, previous results obtained in the rTg4510 mouse model of tauopathy revealed that tangle-bearing neurons respond to novel environments with immediate early gene expression, while in the primary visual cortex tangle-bearing neurons show normal responses to visual stimuli (see Spires-Jones and Hyman, 2014). On the other hand, tangles have been shown to be associated with more subtle neuronal impairments including synapse loss and reduced levels of synaptic vesicle proteins, as well as alterations in the axon initial segment, impaired nucleocytoplasmic transport, and clustering of mitochondria in the soma (Polanco et al., 2018). Assessing the toxicity of tangles is not straightforward and the impact of tangles on neuronal function remains a highly debated yet fundamental question in the field. In this issue of Brain, Marinković and co-workers use longitudinal imaging with in vivo multiphoton microscopy in a model of cortical tangle formation (mutant human tau-overexpressing P301S mice injected with synthetic tau fibrils) to compare the functional properties of tangle-containing versus tangle-free neurons and to track the natural history of these cells (Marinković et al., 2019). This is a powerful experimental approach that makes it possible to visualize directly and with high resolution the longterm functional consequences of newly formed tangles in individual neurons in the living brain (Fig. $1 A)$.

Marinković et al. began by injecting into the frontal cortex of young, pre-tangle P301S mice the genetically encoded calcium indicator GCaMP6s plus synthetic tau fibrils. The latter have been shown to accelerate tangle formation in tau-overexpressing neurons. And indeed, in the tau-injected mice but not in buffer-injected P301S or tau-injected wild-type mice - the authors began to see tangles in vivo about 1 week after the injection. That the tau fibrils instigated the formation of tangles (in a process called tau seeding) was confirmed histologically by tau antibody and silver staining. To 
examine neuronal properties in these mice, the team then imaged spontaneous GCaMP6s calcium signals reflecting action potential firing in both tangle-bearing and tangle-free layer $2 / 3$ cortical neuronal populations. During the imaging the mice were awake in a dark room, either resting quietly or running, without performing any specific behavioural task. Spontaneous activity was suppressed in both tangle-bearing and tangle-free cortical neurons as compared to neurons in wild-type controls (Fig. 1B, left and right panels). Intriguingly, the degree of activity suppression was similar in neurons with and without tangles, suggesting that the tangles did not directly contribute to toxicity. The observation that neurons in pre-tangle P301S mice also had suppressed activity (Fig. 1B, middle panel) reinforced this notion and pointed to a role of soluble tau species in triggering the hypoactivity phenotype. The authors then performed repeated multiphoton imaging of the same neurons for several weeks to assess the consequences of tangles over time, but detected no long-term changes in neuronal activity (Fig. 1C). Finally, by quantifying the pair-wise correlation coefficients for all imaged neurons, the authors examined whether tau-related suppression of activity was associated with an impairment in neuronal population synchrony. Their analysis did not reveal any differences between the tau model and wild-type controls, suggesting that basic local connectivity remained intact. The experiments thus demonstrate that, at least from the perspective of neuronal function, the impact of soluble tau species is greater than that of tangles. This is an important outcome, especially considering that 'tangle busting' compounds are in clinical trials for Alzheimer's disease.

These findings complement and extend previous results from other labs using different tauopathy models and reach similar conclusions, suggesting that the findings are robust and reproducible in multiple settings (Table 1). For example, one study reported a reduction in action potential firing of cortical neurons in young rTg4510 mice with very low tangle burden (Menkes-Caspi et al., 2015). Another study recently showed that suppression of neuronal activity occurs not only in mice that overexpress human tau with disease-causing mutations (e.g., the P301S model used here or rTg4510 mice) but also in mice that overexpress wild-type human tau and essentially never develop any tangles or neurodegeneration (Busche et al., 2019). That study exploited the fact that tau expression could be temporally regulated and showed that turning off tau expression rescued the neuronal hypoactivity phenotype; prior experimental work had already shown that turning off tau expression also recovers memory function and halts neurodegeneration despite the persistence of tangles (Santacruz et al., 2005). The data from these studies and from Marinković and colleagues are based on spontaneous neuronal activity, and it thus remains an open question why tau-containing neurons respond normally to physiological (e.g., visual) stimuli (see Spires-Jones and Hyman, 2014). The observation by Marinković et al. that local neuronal synchrony is preserved may help explain this phenomenon. 
Nevertheless, one would expect the strong decrease in activity to alter neural information processing and the ability to encode new information, e.g. via an increased threshold for induction of synaptic plasticity, which in turn may lead to learning and memory impairments.

The new findings by Marinković et al. raise several additional questions, such as what is the mechanism underlying tau-dependent suppression of neuronal activity? Possible mechanisms include (but are not limited to) pre- and postsynaptic impairments, changes in baseline neuronal excitability, or direct tau effects on transcription in the nucleus. The binding of tau to synaptogyrin-3, for example, reduces synaptic vesicle release (McInnes et al., 2018). Furthermore, tau overexpression is associated with an increased threshold for action potential initiation as well as reduced amounts of AMPA and NMDA glutamatergic receptors at the synapse (Polanco et al., 2018). Notably, mice expressing a rare form of mutated tau (the A152T mutation) exhibit a neuronal hyperexcitability phenotype (Das et al., 2018). Another question is which of the various species of soluble tau are neurotoxic? The race is on to characterize these species as this would likely help to improve the efficacy of anti-tau therapeutics, and hopefully also reduce any impact of these therapies on physiological tau functions. Many experimental studies have relied on the use of tau models that overexpress mutant tau, however growing evidence suggests that wild-type tau also has strong effects on neuronal and vascular functions (Busche et al., 2019). Is that via the same mechanisms as mutant tau? Finally, it is necessary to consider the effects of tau in the context of proteins that co-occur with tau in some tauopathies, in particular Alzheimer's disease. Previous work using various animal models and different experimental settings has revealed that A-beta alone causes neuronal hyperexcitability. Since tau alone suppresses activity, what happens when both proteins are co-expressed? Two recent studies, one in entorhinal cortex slices and the other in cortex in vivo, have provided initial evidence that the combination of tau and A-beta also leads to suppressed activity as the tau phenotype seems to dominate (Angulo et al., 2017; Busche et al., 2019). But this may not be the whole story because there is also strong experimental evidence that under certain circumstances dendritic accumulation of tau can enhance A-beta effects on neurons, and removal of endogenous tau can mitigate a range of A-beta-dependent neuronal impairments (Ittner and Ittner, 2018). Further studies are thus needed to clarify the complex interactions between tau and A-beta.

In conclusion, Marinković et al. show that soluble tau species have greater consequences for neuronal function than insoluble fibrils. They provide powerful evidence that once soluble tau has accumulated in the brain - leading to suppressed neuronal activity - the formation of tangles does not add much 
to the impairments, but neither does it appear to 'protect' neurons from the devastating effects of soluble tau, as had also been hypothesized previously.

\section{Glossary}

P301S model of tauopathy - A transgenic mouse model that overexpresses mutant human P301S tau, which is implicated in familial forms of FTD and parkinsonism linked to chromosome 17 (FTDP-17). The current study used mice at an age ( 2 months) when soluble tau levels are already increased but only a few cortical neurons exhibit tangles.

Tau seeding - Exogenous tau (e.g. pre-formed synthetic tau fibrils) induces formation of tau aggregates and tangles in neurons that express high levels of soluble tau (e.g. those of P301S mice).

GCamP6s - A genetically encoded fluorescent calcium indicator that reliably reports action potential firing in neurons. Here GCaMP6s was co-expressed with the bright structural marker mRuby2, which aids identification of the same cells over repeated imaging sessions.

\section{Figure Legend}

Figure 1: Tau promotes neuronal hypoactivity, even in the absence of tangles. (A) Schematic of the experimental approach of Marinković et al., which combined injection of tau fibrils into the cortex of tau-overexpressing P301S mice with in vivo multiphoton GCaMP6s calcium imaging. Tangle-bearing mice (right panel in B and C) were compared to wild-type mice (left panel) and P301S mice injected with buffer (middle panel). (B) Spontaneous activity of layer $2 / 3$ cortical neurons is suppressed (indicated by blue colour) in tau overexpressing P301S mice with and without tangles (middle and right panels). Further analysis did not detect differences in the activity status of tangle-bearing and tangle-free neurons (right panel). (C) Neuronal activity status remained unchanged over a 10-week recording period.

Table 1: Comparison of neural system changes in various rodent tau models. Data from the present study are shown in rows 1-3, and data from previous studies in rows 4-6.

\section{Competing interest statement}

The author reports no competing interests.

\section{Funding}

This work was supported by the UK Dementia Research Institute. 


\section{Acknowledgements}

Thank you to Bart de Strooper and Hans Förstl for comments on the text.

\section{References}

Angulo SL, Orman R, Neymotin SA, Liu L, Buitrago L, Cepeda-Prado E, et al. Tau and amyloid-related pathologies in the entorhinal cortex have divergent effects in the hippocampal circuit. Neurobiol Dis 2017; 108: 261-76.

Busche MA, Wegmann S, Dujardin S, Commins C, Schiantarelli J, Klickstein N, et al. Tau impairs neural circuits, dominating amyloid-beta effects, in Alzheimer models in vivo. Nat Neurosci 2019; 22(1): 5764.

Das M, Maeda S, Hu B, Yu GQ, Guo W, Lopez I, et al. Neuronal levels and sequence of tau modulate the power of brain rhythms. Neurobiol Dis 2018; 117: 181-8.

Ittner A, Ittner LM. Dendritic Tau in Alzheimer's Disease. Neuron 2018; 99(1): 13-27.

Marinković, P, Blumenstock S, Goltstein P, Korzhova V, Peters F, Knebl A, et al. In vivo imaging reveals reduced activity of neuronal circuits in a mouse tauopathy model. Brain 2019.

Mclnnes J, Wierda K, Snellinx A, Bounti L, Wang YC, Stancu IC, et al. Synaptogyrin-3 Mediates Presynaptic Dysfunction Induced by Tau. Neuron 2018; 97(4): 823-35 e8.

Menkes-Caspi N, Yamin HG, Kellner V, Spires-Jones TL, Cohen D, Stern EA. Pathological tau disrupts ongoing network activity. Neuron 2015; 85(5): 959-66.

Polanco JC, Li C, Bodea LG, Martinez-Marmol R, Meunier FA, Gotz J. Amyloid-beta and tau complexity - towards improved biomarkers and targeted therapies. Nat Rev Neurol 2018; 14(1): 22-39.

Santacruz K, Lewis J, Spires T, Paulson J, Kotilinek L, Ingelsson M, et al. Tau suppression in a neurodegenerative mouse model improves memory function. Science 2005; 309(5733): 476-81.

Spires-Jones TL, Hyman BT. The intersection of amyloid beta and tau at synapses in Alzheimer's disease. Neuron 2014; 82(4): 756-71. 

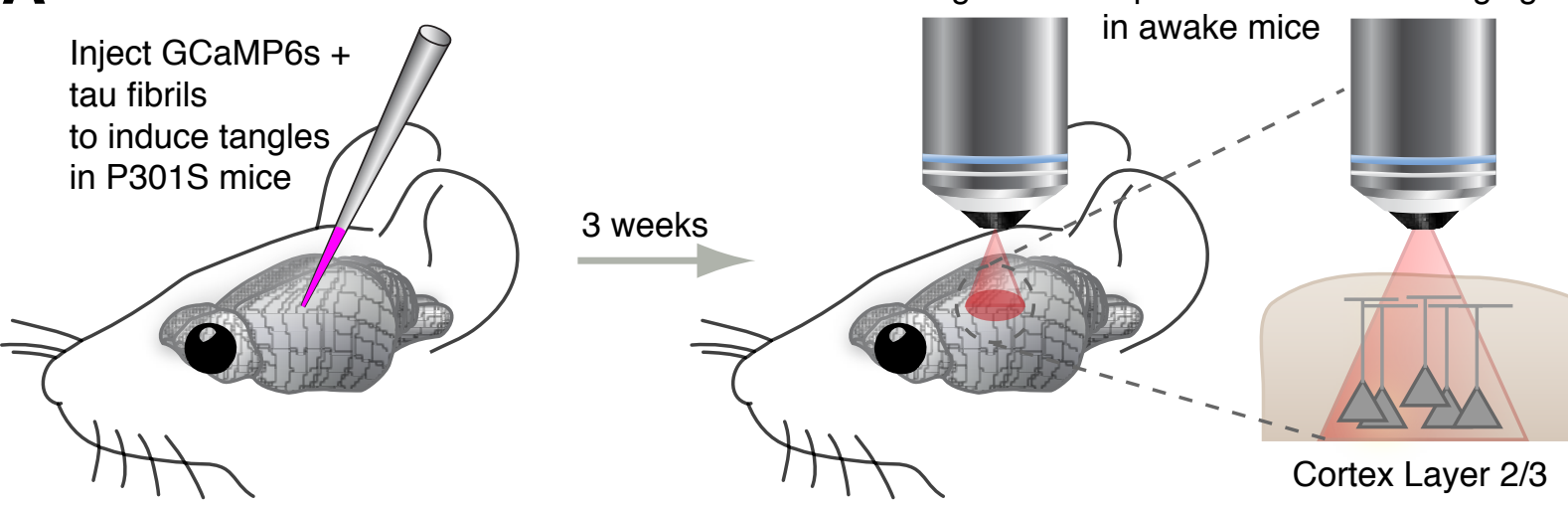

B

Wild-type control

P301S, no tangles (buffer-injected)
P301S, tangles

(fibrils-injected)
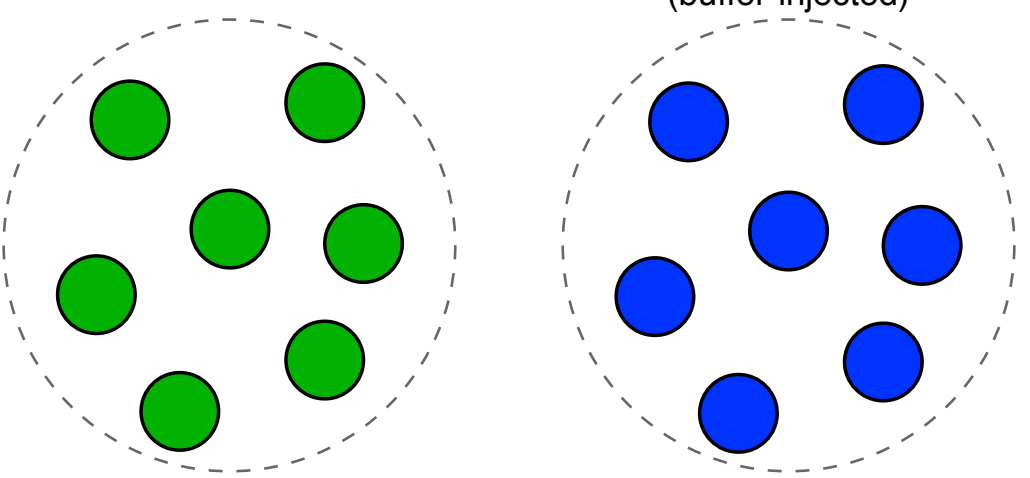

Imaging of the same neurons after 10 weeks

C
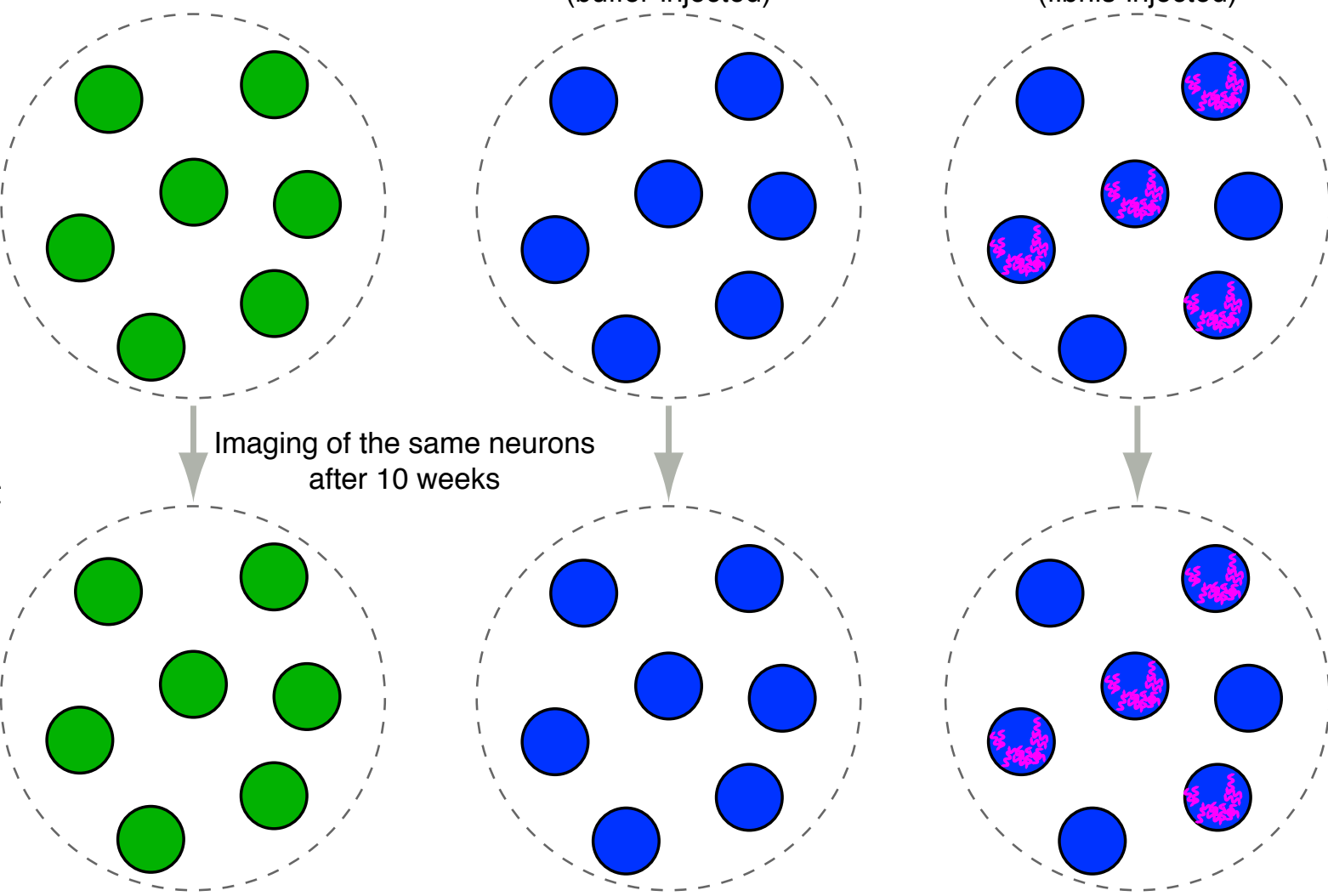

$$
\bigcirc \text { Neuron }
$$

Neuronal activity state 